\title{
Retained Intraspinal Bullet: A Rare Case in a Visitor to Malaysia - A Case Report
}

\author{
MH Ariffin, MS Orth (UKM), Noreen F, MD (UKM), Nor Hamdan Y, MS Orth (UKM), \\ Shaharuddin A R, MS Orth (UKM), Azmi B, MS Orth (UKM) \\ Department of Orthopaedics and Traumatology, Universiti Kebangsaan Malaysia Medical Centre, Kuala Lumpur, \\ Malaysia
}

\begin{abstract}
Firearm injuries to the spine commonly present with acute neurology caused by direct penetration or indirectly from concussive effects of bullet impact on the vertebral column. We report a case of delayed neurologic presentation of retained intra-spinal bullet in a 42 year-old African who had chronic low back pain and sciatica with a past history of gunshot injury to the spine and radiograph revealed a bullet at the L4-5 disc level. Intra-operative exploration showed a fibrous mass around the bullet compressing on the L4 existing nerve root and L5 traversing nerve root. Removal of the retained bullet resulted in a good clinical outcome with complete resolution of symptoms.
\end{abstract}

Key Words:

Intra-spinal bullet, gunshot injury

\section{INTRODUCTION}

We present a case of delayed presentation of neurological deficit in a gunshot injury to the spine; such cases are rare and seldom reported in Malaysia. A 42-year-old African male presented with complaints of chronic low back pain with left leg radiculopathy. He disclosed a history of gunshot injury to the spine upon discovery of a back wound during clinical examination. Radiological examination showed a bullet at the L4/5 disc area. Surgical removal and exploration showed a fibrous mass around a bullet compressing on the left L4 exiting nerve root and the traversing L5 nerve root explaining his symptoms. Surgical removal of the retained bullet and adhesiolysis of the nerve compressed by the fibrous tissue resulted in complete resolution of the patient's symptoms. The presentation of this rare case is highlighted to increase awareness among surgeons especially when treating patients from geographical areas where incidence of gun shot injury are high.

\section{CASE REPORT}

A 42-years-old African male, vacationing in Malaysia, presented to us with a history chronic low back pain associated with shooting pain down the left calf and foot with corresponding numbness on the lateral aspect of the left leg for the past 3 weeks. He had no complaints of weakness and his bowel and bladder is intact. Clinical examination showed that the patient listed to the right and had a scar healed by secondary intention measuring $1.5 \times 1.5 \mathrm{~cm}$ at the left lumbar region $2 \mathrm{~cm}$ from the midline spinous process. The paraspinal muscles were prominent and tender, and upon further enquiry the patient revealed a history of gunshot injury to his back that occurred 9 years ago (for which he had not sought any medical treatment). The gun shot wound had healed with traditional treatment. There was no history to suggest chronic lead poisoning. Neurologic examination revealed paraesthesia of the left L4 and L5 nerve root with no motor weakness. Knee and ankle reflexes were normal. The straight leg raising test (SLR) was positive on the left side. Per-rectal examination was normal.

Radiographic examinations revealed a radiopaque object (bullet fragment) located below the pedicle of L4 just above the disc of L4/5 (Figure 1). There was no fracture seen in radiographs. Computed tomography (CT) imaging further revealed the bony anatomy and exact location of the bullet (Figure 2).

A hemi-laminectomy and left facetectomy revealed a fibrous mass encasing the bullet fragment at the axilla of the left L4 nerve root (Figure 3 ). The fibrous mass was compressing on the left L4 exiting nerve root and the traversing L5 nerve roots explaining the patient's symptoms. Adhesions surrounding the roots were carefully released before removal of retained bullet fragment and instrumented posterolateral fusion was performed. Post-operatively, he had complete resolution of symptoms.

\section{DISCUSSION}

The incidence of civilian gunshot wounds to the spine has been steadily increasing and, in urban areas, it is presently the second leading cause of spinal cord injury. Firearm injuries to the spine may cause damage to the neurological structures by direct penetration or indirectly from concussive 


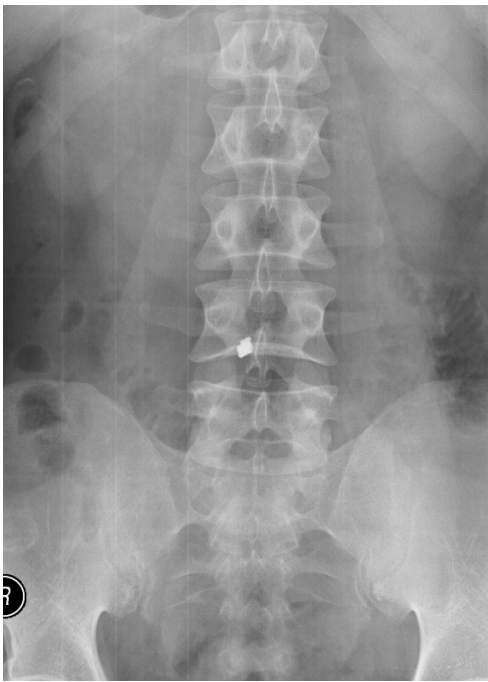

Fig. 1: Lumbosacral anteroposterior radiographic view of the spine showing the bullet at the $L 4 / 5$ area.

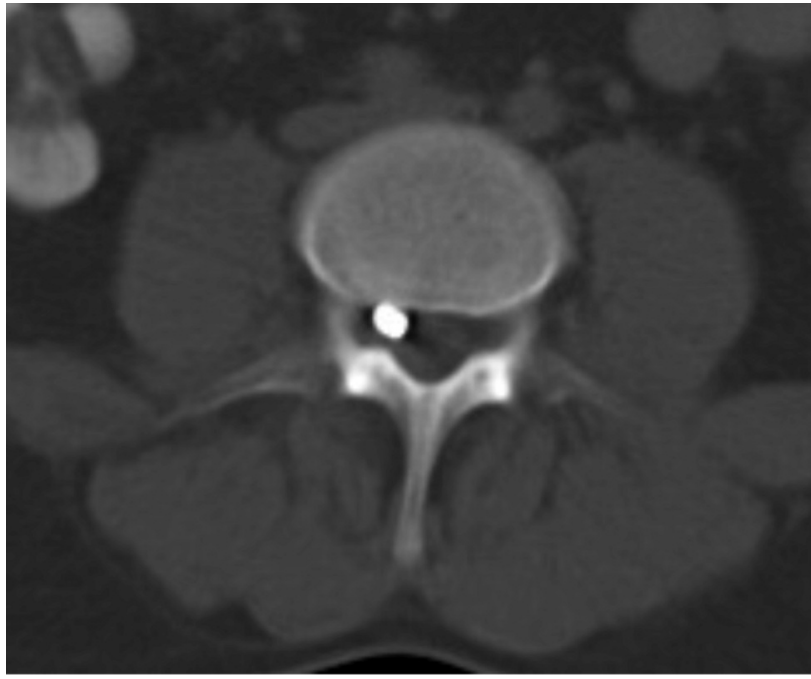

Fig. 2: Axial computed tomography (CT) scan showing location of the bullet in the spinal canal.

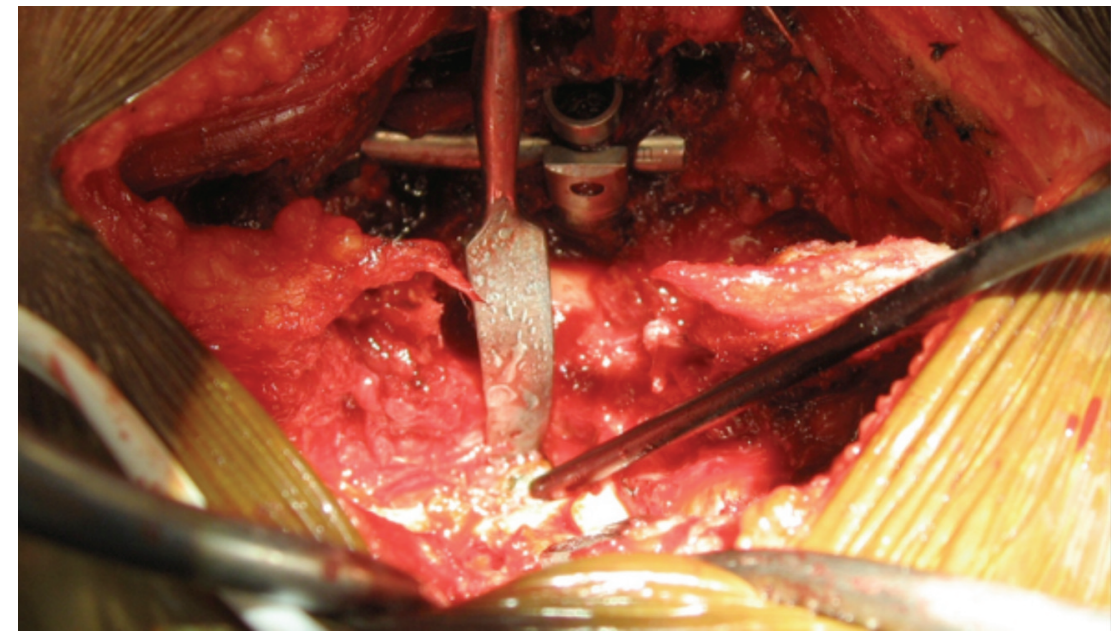

Fig. 3: Intraoperative photograph after complete left $14 / 5$ facetectomy and hemi laminectomy. The dura and the nerve roots were retracted medially to expose the fibrous mass encasing the bullet (pointed out by black suction tip)

effects of the impact of the bullet on the vertebral column. Acute cases of firearm injury (and even more so, chronic retained intraspinal bullets) are rare in Malaysia with only four acute cases of spine gun shot injury recorded in our institution over the past five years.

Plain radiographs are invaluable in determining the level of the bullet, the location of the bullet with respect to the spinal and neural structures and evidence of fractures. Serial radiographs when available will help to determine whether there was any evidence of bullet migration ${ }^{1}$. CT imaging is the advanced modality of choice for further evaluation of spinal gun shot injury. CT images provide a better view of the fracture, a more detailed location of bullet and should reveal evidence of bullet fragmentation. However, CT images may be obscured by artefact when metallic bullet fragments are present ${ }^{1}$, thereby limiting the view and the ability to evaluate fine details.

The role of magnetic resonance imaging (MRI) in evaluating gunshot wound is still controversial because of the fear of bullet migration and further neurological or soft tissue damage from the strong magnetic pull. In a case report by Bashir et al., MRI allowed identification of abnormalities of spinal cord but the case that was presented did not have any retained intraspinal bullet ${ }^{2}$.

Optimal treatment for a bullet lodged in the spinal canal is a matter of controversy. Removal of an intraspinal bullet has been suggested to prevent complications like cerebrospinal fluid leak, meningitis, lead toxicity, back pain or worsening or late onset neurological deficit due to bullet fragment 
migration or the formation of reactive fibrosis ${ }^{1}$. Surgical intervention should be undertaken for patients presenting with neurologic symptoms. Removal of an intraspinal bullet has a positive effect on neurologic recovery when the level of spinal involvement is between T12 and L4 but there is no significant effect on motor recovery in patients with bullet at level between T1 to T11 ${ }^{1}$. Surgical stabilization is indicated in patients with instability of the spine.

Late development of neurological deficit after gunshot injuries to the spine is rare. To our knowledge, very few cases of delayed neurological effects in the form of claudication or radiculopathy from an intraspinal bullet have been reported since $1960^{2-5}$. The timeframe for such presentation ranges from 5 months to 17 years after the gun shot injury. In the case presented here, the patient developed neurological deficit 9 years after the injury.
Previous authors have reported development of dense fibrous tissue, epidural fibrosis and scarring similar to the present case as the cause of delayed neurological presentation ${ }^{2,3}$. In patients without initial neurological deficit, the bullet alone is typically not the cause of the neurological symptoms; instead an extensive chronic inflammatory reaction stimulated by the presence of the bullet is the actual cause of such symptomology ${ }^{3}$. Dense fibrosis surrounding the bullet will ultimately cause spinal stenosis and hence the delayed neurological presentation from central or foraminal stenosis. Removal of the scar and adhesiolysis around the nerve roots results in symptomatic neurological improvement.

\section{REFERENCES}

1. Walters RL, Adkins RH. The effects of removal of bullet fragments retained in the spinal canal. Spine. 1991; 16(8): 934-9.

2. Nino HE, Leppik IL, Lai C, Martin S. Progressive sensory loss one year after bullet injury of spinal cord. JAMA 1978; 240: 11734.

3. Kuijlen JM, Herpers MJ, Beuls EA. Neurogenic claudication, a delayed complication of retained bullet. Spine. 1997; $22(8)$ : $910-4$.

4. Wu WQ. Delayed effects from retained foreign bodies in the spine and spinal cord. Surg Neurol 1986; 25: 214-8.

5. Saad A, Syed AE, Muhammad S. Neurogenic claudication and radiculopathy as delayed presentations of retained spinal bullet. Spine Journal. 2009; 9: 5-8. 